\title{
Análise dos padrões dos movimentos oculares em tarefas de busca visual: efeito da familiaridade $e$ das características físicas do estímulo
}

\author{
Analysis of the eye movement patterns in visual search tasks: \\ effect of familiarityand stimulus features
}

\author{
Elizeu Coutinho de Macedo ${ }^{1}$ \\ Priscila Covre $^{2}$ \\ Fernanda Tebexreni Orsati ${ }^{3}$ \\ Maira Okada de Oliveira ${ }^{4}$ \\ JoséSalomão Schwartzman ${ }^{5}$
}

\begin{tabular}{|l|}
\hline RESUMO \\
\hline Objetivos: Analisar os movimentos oculares numa tarefa de busca visual \\
assimétrica com letras normais e espelhadas. Explorar o efeito da familiari- \\
dade e características do estímulo. Métodos: Oitenta e três universitários \\
emétropes ou com correção óptica foram solicitados a procurar por uma \\
letra normal ou espelhada, num contexto de letras espelhadas ou normais, \\
respectivamente. Foram utilizados quatro tipos de letras (Z, N, E e G) e \\
os movimentos dos olhos foram rastreados por meio de um sistema \\
computacional especializado (eyetracking). As medidas utilizadas para \\
análise foram: tempo de reação, duração e número de fixaçôes, duração \\
e distância dos movimentos sacádicos. Resultados: A análise em função \\
do tipo de letra mostrou diferenças para todas as medidas. O tempo de \\
reação, o número de fixações e a distância média dos movimentos \\
sacádicos foram maiores para encontrar uma letra normal em um conjunto \\
de letras espelhadas. A duração média das fixações, entretanto, foi menor \\
nessa situação. Houve interação dupla entre espelhamento e tipo de letra \\
para o tempo de reação, duração média e número de fixações. O tempo de \\
reação e o número de fixações apresentam função crescente de acordo \\
com o grau de complexidade do estímulo, com aumento acentuado na \\
situação em que o alvo é a letra normal. A duração das fixações, por sua \\
vez, diminui em função da complexidade do estímulo e da situação. \\
Conclusões: Foi mais fácil encontrar uma letra espelhada entre as \\
normais, que o oposto, sendo que o desempenho também sofreu influên- \\
cia do tipo de letra. Quando o contexto é formado por estímulos não \\
familiares e complexos, a duração média das fixações é menor, indicando \\
um processamento visual com amplitude reduzida. Desta forma, são \\
necessárias mais fixações com menor duração para um contexto não \\
familiar e menos fixações com maior duração, para o familiar. \\
\hline
\end{tabular}

Descritores: Movimentos oculares; Percepção visual; Atenção; Reconhecimento visual de modelos

\section{INTRODUĈ̃̃O}

Quando procuramos por um amigo, uma vaga no estacionamento ou uma chave, estamos realizando uma busca visual. A busca visual é uma das ações mais comuns do nosso dia-a-dia e tem sido amplamente investigada há mais de 30 anos em estudos laboratoriais ${ }^{(1)}$. Em geral, é proposto que o participante procure por um estímulo alvo em meio a estímulos distraidores, sendo o tempo de reação a medida de eficiência mais utilizada. Quanto menor o tempo necessário pra encontrar o alvo, mais eficiente é a busca. 
A eficiência da busca depende tanto das características do estímulo alvo ${ }^{(2)}$, quanto dos distraidores ${ }^{(3)}$. Desta forma, uma busca poderá ser eficiente se uma única característica básica, como cor ou tamanho, diferenciar o alvo dos distraidores e, ainda mais eficiente, se os distraidores forem homogêneos.

Entretanto, cor e tamanho não são as únicas características básicas a distinguirem eficientemente os estímulos alvos dos distraidores. Segundo Wolfe ${ }^{(1)}$, muito trabalho tem sido feito a fim de identificar essas características, havendo consenso principalmente no que diz respeito aos atributos físicos do estímulo: orientação, movimento ou curvatura, além dos citados acima ${ }^{(4)}$.

Entretanto, algumas propriedades abstratas também podem atuar na eficiência da busca, tais como, familiaridade e novidade do estímulo. Assim, é mais fácil encontrar um estímulo desconhecido entre estímulos familiares, que um estímulo familiar entre distraidores desconhecidos. Esse é um exemplo de busca assimétrica, onde a troca de papéis entre o estímulo alvo e o distraidor provoca uma diferença na eficiência da busca ${ }^{(5)}$.

Estudos de busca assimétrica com letras espelhadas ou invertidas e letras normais são os mais utilizados para descrever o efeito da familiaridade e da novidade sobre a eficiência ${ }^{(6-12)}$. No entanto, ainda não há um consenso se a assimetria da busca, nesses casos, ocorre devido à familiaridade dos distraidores, à novidade do alvo ou em função de ambos.

Para alguns autores, o fato de que é mais fácil encontrar letras espelhadas ou invertidas entre letras normais que o contrário, deve-se à familiaridade dos distraidores. Ou seja, as pessoas procuram mais rapidamente em ambientes familiares que em ambientes desconhecidos. A natureza do contexto é mais importante que a do alvo ${ }^{(9-1)}$.

Treisman e Souther ${ }^{(5)}$ propuseram que transformações num estímulo padrão fariam com que este chamasse mais atenção que o próprio estímulo não alterado, ou seja, um estímulo novo, como uma letra espelhada, chamaria mais atenção que um estímulo padrão, uma letra normal. Há uma tendência de um estímulo novo ou inesperado capturar a atenção ${ }^{(13-14)}$. Desta forma, alguns trabalhos atribuem a assimetria da busca de letras espelhadas não somente à familiaridade do fundo, mas também à novidade do estímulo alvo ${ }^{(6-7)}$.

De acordo com Wang et $\mathrm{al}^{(7)}$, a assimetria da busca de letras pode ser mais dramática para alguns pares de letras que para outros, o que indica que além dos fatores de familiaridade e novidade, as características físicas básicas também são importantes para caracterizar a assimetria na eficiência da busca. Um dos objetivos do presente estudo é explorar o efeito do tempo na busca visual para as letras Z, N, E e G em sua posição normal e em suas formas espelhadas: $\mathrm{Ze}, \mathrm{Ne}, \mathrm{Ee}$ e Ge.

A fim de explorar o efeito do contexto e novidade dos estímulos, serão investigados os padrões dos movimentos oculares resultantes da busca visual assimétrica para cada tipo de letra. As variáveis a serem consideradas serão: o tempo de reação para encontrar o estímulo alvo, o número de fixações, a duração média de cada fixação, a distância média dos comprimentos sacádicos e a duração média dos movimentos sacádicos.

\section{MÉTODOS}

Participantes: 83 estudantes universitários, 42 homens $(\mathrm{M}=23,83 ; \mathrm{DP}=3,48$ anos de idade) e 41 mulheres $(\mathrm{M}=22,73$; $\mathrm{DP}=3,51$ anos de idade). Todos os participantes eram emétropes ou com correção óptica.

Equipamento: Para a apresentação dos estímulos e rastreamento dos movimentos oculares foi usado o Sistema Eyegaze ${ }^{\circledR}$ desenvolvido pela LC Technologies, Inc (2001). Este sistema registra os movimentos oculares do participante automaticamente e em tempo real. A direção do olhar é determinada pelo método de reflexo da córnea no centro da pupila (PCCR-PupilCenter-Corneal Reflexion) o que possibilita avaliar usuários com lentes corretivas. O equipamento é calibrado para o olho dominante e as medidas são feitas em uma frequência de amostragem de 60 Hertz. Para cada amostragem, são geradas as seguintes informações: "flag" indicando se a imagem do olho foi capturada pela câmera; diâmetro e posição da pupila a partir das coordenadas x e y do plano da tela do computador; deslocamento do globo ocular ao longo do eixo z; duração e localização das fixações e movimentos sacádicos.

Foi usado um microcomputador com processador Intel Pentium ${ }^{\circledR}$ III, $128 \mathrm{Mb}$ de memória RAM, placa de vídeo de $2 \mathrm{Mb}$, monitor de vídeo com tela plana do tipo LCD (liquid crystal display) de 15 " com resolução de $800 \times 600$ pixels, suporte para monitor de câmera com lentes sensíveis a infravermelho de alta velocidade (RS-170 ou CCIR) e LED (light emitting diode) emissor de raios infra-vermelhos. A luminância média do monitor de vídeo com os quadros de estímulos apresentados era de $85 \mathrm{~cd} / \mathrm{m}^{2}$ e a da sala de avaliação de $4 \mathrm{~cd} / \mathrm{m}^{2}$. Foi utilizada uma mesa de suporte oftalmológico (LF-M4 da DFV S/A) para evitar movimentos da cabeça e manter a distância dos olhos dos participantes a 50 centímetros da tela do computador.

Estímulos: Foram apresentados oito quadros de maneira aleatória para cada sujeito. Os quadros ocupavam toda a tela do computador e eram compostos por quatro quadrantes contendo um mesmo tipo de letra, sendo que em apenas um dos quadrantes era apresentada a letra-alvo normal ou espelhada. As letras utilizadas para a composição dos quadros foram $\mathrm{Z}$, N, E e G. Foram gerados oito quadros diferentes, pois cada letra foi apresentada em duas situações. Na primeira situação, os estímulos distraidores eram letras normais e o estímuloalvo, a letra espelhada. Na segunda, os estímulos distraidores eram as letras espelhadas e o estímulo-alvo, a letra normal. Assim, na primeira situação, o participante deveria encontrar uma das letras espelhadas, a letra espelhada (e) entre as letras normais: Ne-N, Ze-Z, Ee-E, Ge-G. Na segunda, o estímulo a ser encontrado seria a letra normal entre as letras espelhadas: $\mathrm{N}$ $\mathrm{Ne}, \mathrm{Z}-\mathrm{Ze}, \mathrm{E}-\mathrm{Ee}, \mathrm{G}-\mathrm{Ge}$. Os estímulos-alvos foram distribuídos aleatoriamente nos quadros. A figura 1 ilustra um dos quadros apresentados, onde o estímulo alvo era o $\mathrm{N}$ espelhado $(\mathrm{Ne})$ e os estímulos distraidores, a mesma letra em sua forma normal. Neste exemplo, o estímulo alvo está localizado no quadrante inferior à esquerda. 


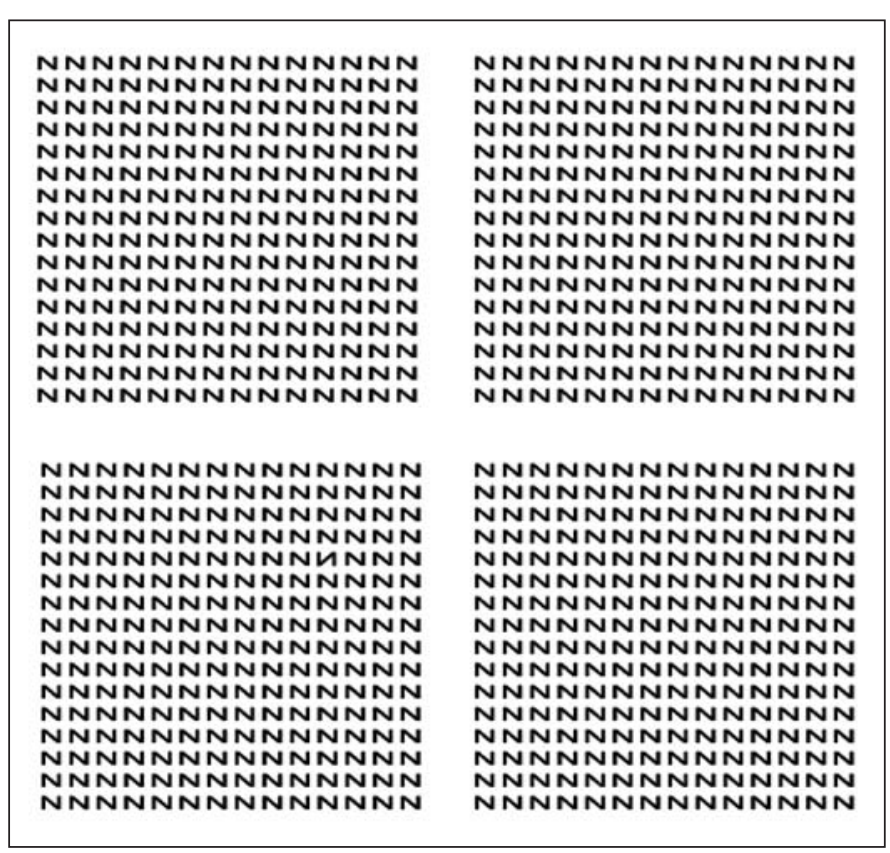

Figura 1 - Quadro Ne-N, o estímulo alvo é a letra $\mathrm{N}$ espelhada $(\mathrm{Ne})$ e os estímulos distratores a letra $\mathrm{N}$ normal. Neste exemplo, o estímulo alvo está localizado no quadrante inferior à esquerda.

Procedimento: A pesquisa foi previamente aprovada pelo Comitê de Ética em Pesquisa da Universidade Presbiteriana Mackenzie. Cada sessão era individual, com a duração média de 15 minutos. Todos os participantes foram avaliados através da escala optométrica de Snellen e deveriam apresentar visão igual ou superior a 0,9 para serem incluídos no estudo. As instruções foram dadas por escrito, contando com um exemplo da tarefa a ser executada, a fim de evitar possíveis dicas dadas pelo examinador. A situação do exemplo era idêntica, em tamanho, à do teste e o participante deveria ser capaz de identificar e encontrar a letra para prosseguir. Aqueles que não foram capazes de encontrar o estímulo alvo dos distratores em até 1 minuto foram excluídos do estudo.

A tarefa consistia em encontrar um estímulo diferente em meio a estímulos iguais. O sujeito era instruído a procurar pelo estímulo alvo no quadro apresentado, o mais rápido possível. Assim que o encontrasse, deveria manter os olhos fixos nele e avisar o examinador que finalizaria a exposição do quadro. Havia um tempo limite de 60 segundos para a busca, ou seja, caso não encontrasse o estímulo, nesse tempo a tela era fechada automaticamente.

Para análise dos resultados foi utilizado o programa de análise estatística SPSS 13.0 e o nível de significância adotado foi de 5\%. Foram conduzidas análises de variância para medidas repetidas (2x4), sendo as variáveis independentes o espelhamento do estímulo alvo e o tipo de letra. As variáveis dependentes foram: o tempo de reação para encontrar o estímulo alvo (TR), o número de fixações (NF), a duração média de cada fixação (DurMF), a distância média dos comprimentos sacádicos (DistMS) e a duração média dos movimentos sacádicos (DurMS).

\section{RESULTADOS}

Os resultados mostraram que, de modo geral, foi mais fácil encontrar uma letra espelhada entre as normais, que o oposto. Assim, análises dos efeitos principais mostraram que, das 5 variáveis dependentes analisadas, 4 apresentaram diferenças significativas em função do espelhamento do estímulo. A tabela 1 sumariza os resultados de média, desvio padrão, o valor do F e a significância (p) para os estímulos-alvo espelhado e normal.

O tempo de reação e o número de fixações foram aproximadamente duas vezes maiores para encontrar uma letra normal em um conjunto de letras espelhadas. A duração média das fixações, por sua vez, foi significativamente menor nessa condição. Pode-se observar que os padrões dos movimentos sacádicos também foram sensíveis ao grau de dificuldade da tarefa, sendo que a duração média das sacadas foi maior na condição mais fácil, e a distância média dos movimentos sacádicos tendeu a ser maior na condição mais fácil.

Desta forma, nas situações difíceis, quando as letras espelhadas são utilizadas como estímulos distraidores, é realizado um maior número de fixações, com menor duração. Nas situações fáceis, o tempo médio de fixação é menor e o comprimento médio da sacada, maior. Observa-se, portanto, uma maior eficiência da busca visual.

Com relação ao tipo de letra que compunha os quadros, foram observadas diferenças significativas. A tabela 2 sumariza os resultados de média, desvio padrão, o valor do $\mathrm{F}$, a significância (p) e análise post hoc (Bonferroni) para cada tipo de letra. Foi observada uma função crescente do tempo de reação e do número de fixação para encontrar o estímulo-alvo e uma função decrescente da duração média das fixações e a distância média dos movimentos sacádicos, na seguinte ordem: Z, N, E e G. Assim, as letras Z e N foram as mais fáceis e as letras E e $\mathrm{G}$, as mais difíceis.

Esses resultados podem ser explicados pelas características físicas que distinguem os estímulos-alvo dos distraídores. Enquanto o espelhamento das letras E e G promove mudança na configuração da letra, alterando a orientação da mesma como um todo, o espelhamento das letras $\mathrm{N}$ e $\mathrm{Z}$ altera somente a angulação da linha oblíqua interna, sem alteração das linhas periféricas. Isto faz com que as letras $\mathrm{N}$ e $\mathrm{Z}$, diante de distraídores espelhados, sejam mais facilmente detectáveis, ainda que haja uma grande discrepância entre os sujeitos como mostrado pelos elevados valores de desvios-padrão. Além disso, características lineares são mais simples e facilmente identificáveis que as curvas, o que torna a letra $\mathrm{G}$ ainda mais complexa que as outras.

Os achados indicam, portanto, que estímulos mais fáceis demandam menos fixações, entretanto a duração e a distância entre elas tendem a serem maiores. Embora não tenham sido encontradas diferenças significativas para a duração média dos movimentos sacádicos, observa-se também uma função linear decrescente.

Foi encontrado também efeito de interação dupla do tipo 


\begin{tabular}{|c|c|c|c|c|}
\hline & $\begin{array}{l}\text { Alvo normal } \\
\text { Média (DP) }\end{array}$ & $\begin{array}{l}\text { Alvo espelhado } \\
\text { Média (DP) }\end{array}$ & $\mathbf{F}$ & $\mathbf{p}$ \\
\hline TR (ms) & $11083,76(8074,86)$ & $5321,18 \quad(4492,23)$ & 34,333 & 0,000 \\
\hline NF & $29,82(19,45)$ & $15,39(11,40)$ & 34,733 & 0,000 \\
\hline DurMF (ms) & $403,18(81,74)$ & $458,97(133,34)$ & 12,074 & 0,001 \\
\hline DistMS (graus) & $3,54(\quad 0,57)$ & $3,70($ & 3,771 & 0,056 \\
\hline DurMS (ms) & $23,46(21,89)$ & $30,14(23,31)$ & 7,262 & 0,009 \\
\hline
\end{tabular}

\begin{tabular}{|c|c|c|c|c|c|c|c|}
\hline & $\begin{array}{c}Z \\
\text { Média (DP) }\end{array}$ & $\begin{array}{c}\mathrm{N} \\
\text { Média (DP) }\end{array}$ & $\begin{array}{c}E \\
\text { Média (DP) }\end{array}$ & $\begin{array}{c}\mathrm{G} \\
\text { Média (DP) }\end{array}$ & $F$ & $p$ & \\
\hline TR & $3,52(5,66)$ & $5,54(6,56)$ & $7,04(5,61)$ & $17,57(10,95)$ & 56,630 & 0,000 & $a, b, c, e, f$ \\
\hline NF & $9,35(12,47)$ & $15,50(16,68)$ & $20,01 \quad(13,64)$ & $47,87(27,06)$ & 67,780 & 0,000 & $a, b, c, e, f$ \\
\hline DurMF & $517,60(188,13)$ & $449,81 \quad(134,28)$ & $405,97(177,39)$ & $357,11(57,72)$ & 15,759 & 0,000 & $a, b, c, e$ \\
\hline DistMS & $4,05(1,25)$ & $3,52(0,89)$ & $3,64(0,71)$ & $3,19(0,56)$ & 13,570 & 0,000 & $a, b, c, e, f$ \\
\hline DurMS & $30,88(26,05)$ & $27,72(23,64)$ & $25,97 \quad(23,87)$ & $22,21 \quad(32,21)$ & 2,031 & 0,111 & $c, e$ \\
\hline
\end{tabular}

de letra e espelhamento do estímulo para tempo de reação $\left(\mathrm{F}_{[3,129]}=4,440 ; \mathrm{p}=0,005\right)$, número de fixações $\left(\mathrm{F}_{[3,129]}=5,233\right.$; $\mathrm{p}=0,002)$ e duração média das fixações $\left(\mathrm{F}_{[3,129]}=3,020 ; \mathrm{p}=0,032\right)$. Não foram encontradas interações para as medidas de movimentos sacádicos.

Os gráficos 1 e 2 ilustram, respectivamente, a interação do tempo de reação e do número de fixações em função do grau crescente de complexidade da letra e do espelhamento do estímulo. Nota-se uma função crescente, sendo mais acentuada para a condição mais difícil. A duração média das fixações, entretanto, decresce de acordo com a complexidade da tarefa.

Assim, para os estímulos mais simples, o estímulo alvo é encontrado com um pequeno número de fixações, mas as

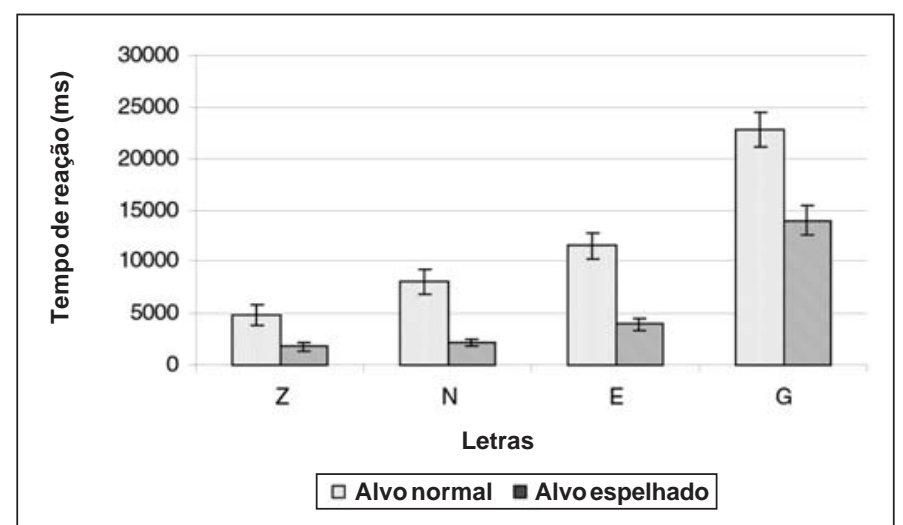

Gráfico 1 - Tempo de reação em função do tipo de letra e da relação estímulo-alvo e distraidor: encontrar a letra normal entre os distraidores espelhados (alvo normal) e encontrar a letra espelhada entre os distraidores normais (alvo espelhado) durações médias destas fixações são altas. À medida que o grau de complexidade do estímulo aumenta, o número de fixações necessárias para encontrar o estímulo alvo aumenta e a duração média das fixações diminui. Sendo observado o mesmo tempo médio nas fixações para a situação $G$, independentemente da relação entre estímulo alvo e distraidores.

A fim de analisar o efeito do sexo sobre o desempenho na tarefa de busca visual, foram conduzidos teste $t$ de medidas independentes para cada uma das variáveis. Não foram encontradas diferenças entre homens e mulheres para nenhuma das variáveis analisadas: tempo de reação, número de fixações, duração média das sacadas, tempo e comprimento médio dos movimentos sacádicos.

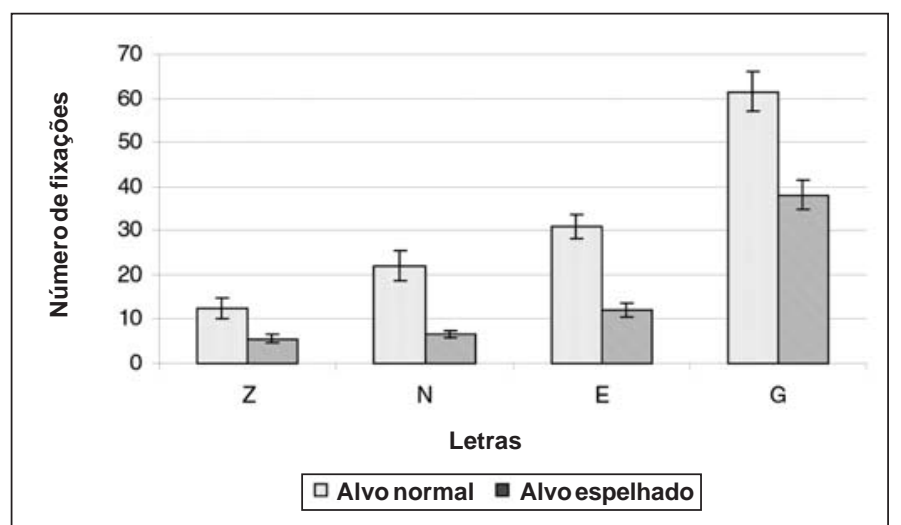

Gráfico 2. Número de fixações em função do tipo de letra e da relação estímulo-alvo e distraidor: encontrar a letra normal entre os distraidores espelhados (alvo normal) e encontrar a letra espelhada entre os distraidores normais (alvo espelhado) 


\section{DISCUSSÃO}

O tempo de reação é a medida mais utilizada para avaliar o desempenho em tarefas de busca visual. Assim, um menor tempo corresponde a um melhor desempenho. Numa situação de busca assimétrica, a inversão de papéis entre o estímuloalvo e os estímulos distraidores, provoca uma grande diferença no desempenho, o que pode ser analisado em função da familiaridade do estímulo ${ }^{(6-12)}$. O efeito de assimetria foi encontrado nesse estudo, sendo mais fácil encontrar uma letra espelhada entre as letras em sua forma normal, que o oposto.

O desempenho também sofreu influência do tipo de letra utilizado, corroborando os achados de Wang et al ${ }^{(7)}$. Desta forma, foi mais difícil encontrar letras com características curvas, como o G, que lineares, como o N, Z e E. Além disso, quando o efeito de assimetria ocorreu pela distinção da direção de uma linha oblíqua, como o $\mathrm{N}$ e Z, a busca foi mais fácil que quando ocorreu por uma distinção na orientação, como $\mathrm{E}$ e G. Estes achados podem ser explicados pela teoria das características $^{(2)}$, que propõe que a facilidade de encontrar um alvo depende de características primitivas dos estímulos.

Em suma, o desempenho nas tarefas de busca visual foi função tanto da familiaridade do estímulo, quanto de suas características $^{(4)}$.

A fim de compreender o processamento envolvido nesta diferença de desempenho, foram analisadas medidas dos movimentos oculares. Segundo Just e Carpenter ${ }^{(15)}$, a partir da análise do padrão da fixação dos olhos pode-se inferir a respeito da estratégia utilizada em tarefas cognitivas. Ou seja, um processamento efetivo depende da duração da fixação ocular e varia de acordo com as características do estímulo ${ }^{(16)}$.

Greene e Rayner ${ }^{(17)}$ propuseram a hipótese da amplitude de processamento para explicar o que ocorre com a fixação ocular diante de estímulos familiares e não familiares. De acordo com essa hipótese, quando os estímulos são familiares, o observador pode adquirir mais informações através de uma amplitude de processamento maior, assim, as fixações duram mais e o estímulo é encontrado mais facilmente. Para estímulos não familiares, porém, a amplitude é mais estreita, sendo necessário um número maior de fixações com durações menores até que o alvo seja encontrado.

Os resultados de Greene e Rayner ${ }^{(17)}$ foram reproduzidos nesse estudo, corroborando a hipótese da amplitude de processamento. Ou seja, nas condições mais difíceis, foi feito um número maior de fixações oculares com menor duração. Entretanto, no presente trabalho, a diferença na amplitude de processamento dependeu não somente da familiaridade dos estímulos, mas também das características físicas dos mesmos. Assim sendo, estímulos mais facilmente processados (i.e.: letra Z) contaram com uma maior amplitude de processamento, que os mais difíceis (i.e.: letra $\mathrm{G}$ ).

Além disso, tarefa de busca visual proposta neste estudo é mais complexa. Os quadros apresentados contaram com um número significativamente maior de distraidores sendo, portanto, uma tarefa mais complexa. Apesar disso, o efeito da amplitude de processamento foi encontrado, demonstrando consistência na hipótese apresentada pelos autores. Este achado é importante, pois o uso de tarefas mais complexas de busca visual teve como objetivo criar uma condição mais próxima das situações naturalísticas. Uma outra forma utilizada para garantir a consistência do efeito foi a avaliação de um número grande de sujeitos.

Em suma, é mais fácil encontrar uma letra espelhada entre as normais, que o oposto, sendo que o desempenho também sofre influência do tipo de letra. A análise dos movimentos oculares aponta para diferenças no processamento ocular nas condições fáceis ou difíceis. A diminuição da duração média das fixações nas situações difíceis pode indicar que, quando o contexto é formado por estímulos complexos, o processamento visual tenha uma amplitude reduzida. Desta forma, são necessárias mais fixações oculares com menor duração para um contexto de distraidores complexos. E, menos fixações com maior duração, para os distraidores mais simples.

\section{ABSTRACT}

Purpose: To analyze eye movements in asymmetric visual search using the task of normal and mirrored position letters. To evaluate the effect of familiarity and stimulus features. Methods: Eighty-three university students with normal or corrected-to-normal vision were asked to search for a letter in inverted position to the letters in a group of either normal or mirrored letters. Four types of letters were used (Z, N, E and G) and the eye movements were tracked by a specialized computer-based system (eyetracking). The analyzed measurements were: reaction time, fixation number and duration, saccade distance and duration. Results: All measures varied with the type of letter. Reaction time, fixation number, and saccade distance were higher when the task was to find the normal letter in a group of mirrored letters. In this condition, fixation duration was smaller. Interaction was found between familiarity and the type of letter for the reaction time, fixation number and duration. The reaction time and fixation number increased together with the stimulus complexity, with a greater increase for the normal letter target. Fixation duration, however, decreased with the complexity of the stimuli and the search condition. Conclusions: Finding a mirrored letter among normal letters proved to be easier than the contrary. The letter type also affected the performance. When the context is formed of unfamiliar complex stimuli, the fixation duration is shorter, indicating a narrower span for visual processing. Therefore, a greater number of fixations with shorter duration are needed for the unfamiliar context while less fixations with greater duration are needed for the familiar context.

Keywords: Eye movements; Visual perception; Attention; Pattern recognition, visual 


\section{REFERÊNCIAS}

1. Wolfe JW. Asymmetries in visual search: an introduction. Percept Psychophys. 2001;63(3):381-9.

2. Treisman AM, Gelade G. A feature-integration theory of attention. Cognit Psychol. 1980;12(1):97-136.

3. Duncan J, Humphreys GW. Visual search and stimulus similarity. Psychol Rev. 1989;96(3):433-58.

4. Wolfe JM. Visual Search. In: Pashler H, editor. Attention. London: Psychology Press; 1998. p.13-73.

5. Treisman A, Souther J. Search asymmetry: a diagnostic for preattentive processing of separable features. J Exp Psychol Gen. 1985;114(3):285-310.

6. Malinowski P, Hübner R. The effect of familiarity on visual-search performance: evidence for learned basic features. Percept Psychophys. 2001;63(3): 458-63.

7. Wang Q, Cavanagh P, Green M. Familiarity and pop-out in visual search. Percept Psychophys. 1994;56(5):495-500.

8. Ambler BA, Proctor JD. The familiarity effect for single-letter pairs. J Exp Psychol Hum Percept Perform. 1976;2(2):222-34.
9. Shen J, Reingold EM. Visual search asymmetry: the influence of stimulus familiarity and low-level features. Percept Psychophys. 2001;63(3):464-75.

10. Greenberg SN, Krueger LE. Effect of letter orientation and sequential redundancy on the speed of letter search. Mem Cognit. 1983;11(2):181-91.

11. Reicher GM. Familiarity of background characters in visual scanning. J Exp Psychol Hum Percept Perform. 1976;2(4):522-30.

12. Frith U. A curious effect with reversed letters explained by a theory of schema. Percept Psychophys. 1974;16(1):113-6.

13. Johnston WA, Hawley KJ, Farnham JM. Novel popout: empirical boundaries and tentative theory. J Exp Psychol Hum Percept Perform. 1993;19(1):140-53.

14. Hawley KJ, Johnston WA, Farnham JM. Novel popout with nonsense strings: effects of predictability of string length and spatial location. Percept Psychophys. 1994;55(3):261-8.

15. Just MA, Carpenter PA. Eye fixations and cognitive processes. Cognit Psychol. 1976;8:441-80.

16. Bertera JH, Rayner K. Eye movements and the span of the effective stimulus in visual search. Percept Psychophys. 2000;62(3):576-85.

17. Greene HH, Rayner K. Eye movements and familiarity effects in visual search. Vision Res. 2001;41(27):3763-73.

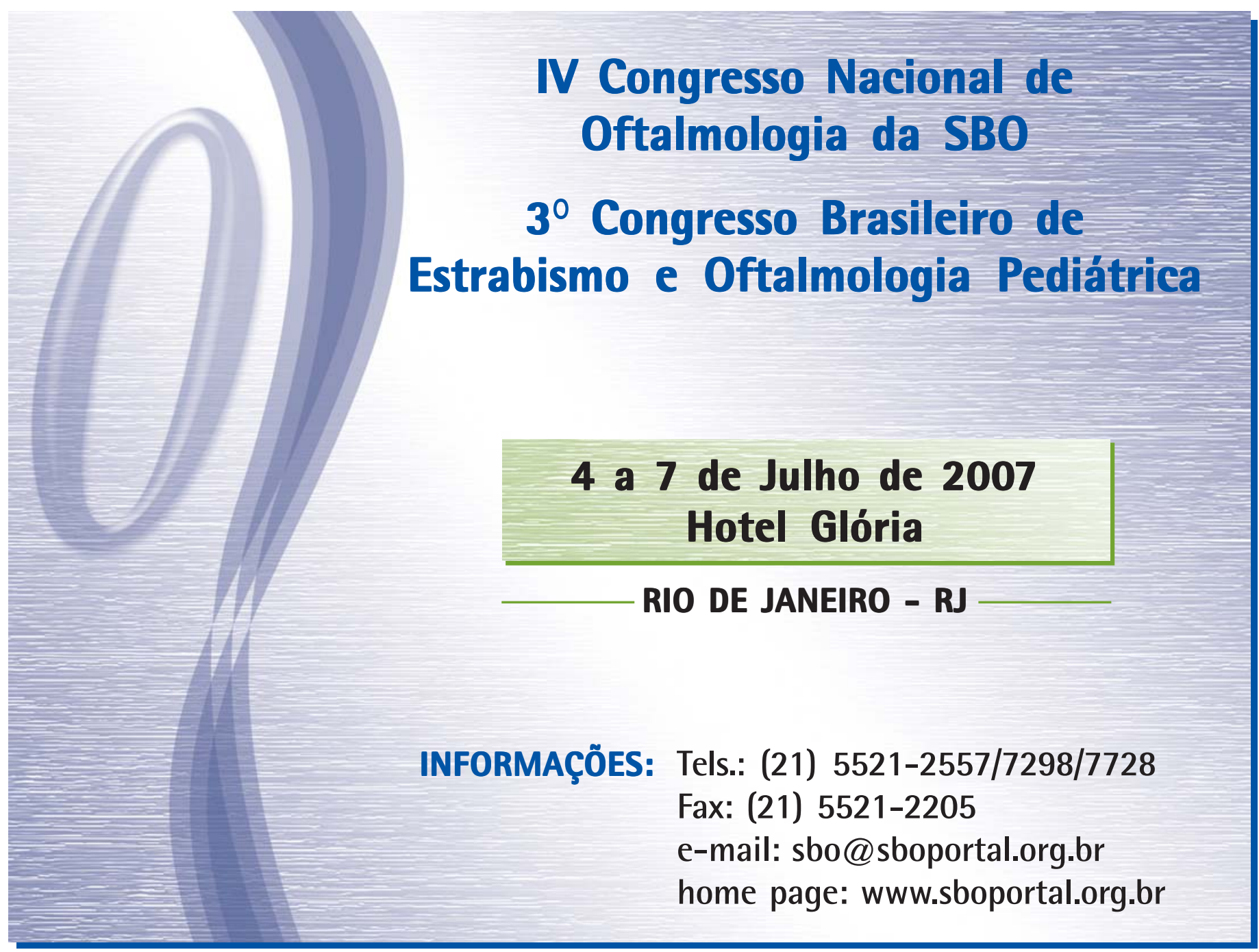

